\title{
Fermions in higher representations. Some results about SU(2) with adjoint fermions.
}

\section{Luigi Del Debbio}

SUPA, School of Physics and Astronomy, University of Edinburgh, Edinburgh EH9 3JZ, Scotland

E-mail: luigi.del.debbioded.ac.uk

\section{Agostino Patella*}

Department of Physics, Swansea University, Singleton Park, Swansea SA2 8PP, Wales

E-mail: a.patella@swansea.ac.uk

\section{Claudio Pica}

Physics Department, Brookhaven National Laboratory, Upton, NY 11973-5000, USA

E-mail: pica@quark.phy.bnl.gov

\begin{abstract}
We discuss the lattice formulation of gauge theories with fermions in arbitrary representations of the color group, and present the implementation of the RHMC algorithm for simulating dynamical Wilson fermions. A first dataset is presented for the $\mathrm{SU}(2)$ gauge theory with two fermions in the adjoint representation, which has been proposed as a possible technicolor candidate. Simulations are performed on $8^{3} \times 16$ lattices, at fixed lattice spacing. The PCAC mass, the pseudoscalar, vector and axial meson masses, the pseudoscalar meson decay constant are computed. The extrapolation to the chiral limit is discussed. However more extensive investigations are needed in order to control the systematic errors in the numerical results, and then understand in detail the phase structure of these theories.
\end{abstract}

The XXVI International Symposium on Lattice Field Theory

July 14 - 19, 2008

Williamsburg, Virginia, USA

\footnotetext{
* Speaker.
} 


\section{Introduction}

Gauge theories with fermions in two-index representations play important roles in different scenarios for Physics beyond the Standard Model (SM): supersymmetric theories, technicolor models $[1,2,3,4]$, orientifold theories [5, 6]. So far the lattice is the unique tool for studying stronglyinteracting theories from first principles. Moreover recent algorithmic progress and the increase in computer performance allow these theories to be studied by Monte Carlo simulations at an affordable cost. On the other hand the expertise developed in many years of simulating lattice QCD must be exported with some care as we try to interpret results for generic strongly-interacting theories, since these may reveal a very different phenomenology from QCD.

Therefore the challenge this work attempts to address is twofold. We generalize the standard RHMC algorithm for simulating QCD to the case of $\mathrm{SU}(N)$ gauge group and fermions in a generic representation. We briefly describe the main characteristics of our bespoke code - HiRep - in which we have implemented the two-index representations for generic number of colors. Then we present some preliminary result for $\mathrm{SU}(2)$ with two Dirac fermions in the adjoint representation.

The number of fermions in this theory is close to the estimated critical value where an infrared (IR) fixed point is expected to appear [7]. If an IR fixed point exists, the theory is conformal in the IR, in particular it does not confine and does not break chiral symmetry. If the theory does not have the IR fixed point, the vicinity to the critical number of flavours can induce a walking behaviour of the renormalized coupling constant, which would make such a theory a possible realization of the technicolor mechanism $[8,9]$. Which scenario is the correct one can only be established by numerical simulations on the lattice. However for realistic numerical simulations, the finite volume, the non-zero fermion mass, and the lattice artifacts introduce systematic errors that are likely to obscure the behaviour one would naively infer from the existence of an IR fixed point. In order to obtain clear and robust results, we need to go to large volumes, small masses and small lattice spacing, keeping in mind that we do not know a priori what large and small mean in this case.

Presenting the results, we will try to emphasize which are compatible with a standard QCDlike scenario, and which deviate from it giving hints of new interesting dynamics.

At the end of this introduction, we want to point out that the same theory was explored in [10]; other gauge theories close to the conformal window have recently been investigated in [11, 12, 13] (see also the talks of De Grand, Deuzeman, Fleming, Hietanen, Holland, Jin, Neil, Nogradi, Pallante, Svetitsky in this conference).

\section{Description of the HiRep code}

The HiRep code implements the RHMC algorithm [14] for simulating dynamical Wilson fermions in a generic representation of $\mathrm{SU}(N)$, once the mathematical definition of the representation is provided. One and two-index representations are implemented. In what follows, we summarize the main features of the implementation (for details, see [15]).

Generic representations. We call $T^{a}$ the generators of the $\mathrm{SU}(N)$ group, $T_{R}^{a}$ the represented generators in the representation $R$, and $T_{R}$ their normalization defined as $\operatorname{tr}\left[T_{R}^{a} T_{R}^{b}\right]=T_{R} \delta_{a b}$. For 
each link, we store both the link-variable in the fundamental representation $U(x, \mu)$ and the represented one, defined by:

$$
U_{R}(x, \mu)=\exp \left[i \omega^{a}(x, \mu) T_{R}^{a}\right], \quad \text { with } \quad U(x, \mu)=\exp \left[i \omega^{a}(x, \mu) T^{a}\right] .
$$

In the code, the generators $T^{a}, T_{R}^{a}$ an the definition of $U_{R}$ are implemented for one and twoindex representations.

Dirac operator. The Wilson discretization of the Dirac operator is obtained from the naive one by simply replacing the link-variables with the represented ones:

$$
D \psi(x)=\psi(x)-\kappa \sum_{\mu}\left\{\left(1-\gamma_{\mu}\right) U_{R}(x, \mu) \psi(x+\hat{\mu})+\left(1+\gamma_{\mu}\right) U_{R}(x-\hat{\mu}, \mu)^{\dagger} \psi(x-\hat{\mu})\right\} .
$$

We also define the Hermitian operator $Q=\gamma_{5} D$.

MD Hamiltonian. The determinant of the Dirac operator in the partition function is stochastically estimated:

$$
\operatorname{det} D^{n_{f}}=\operatorname{det} Q^{n_{f}} \propto \int \exp \left\{-\phi^{\dagger} Q^{-n_{f}} \phi\right\} \mathscr{D} \phi \mathscr{D} \phi^{\dagger},
$$

and the generic negative powers of $Q^{2}$ are computed by means of rational approximations $x^{-p / 2} \simeq \sum_{i} \alpha_{i}^{(p)} /\left(x-\beta_{i}^{(p)}\right)$,

$$
Q^{-n_{f}}=\left(Q^{2}\right)^{-n_{f} / 2} \simeq \sum_{i} \alpha_{i}^{\left(n_{f}\right)}\left[Q^{2}-\beta_{i}^{\left(n_{f}\right)}\right]^{-1}
$$

The inversions in the expressions above are performed simultaneously thanks to the implementation of a multi-shift inverter. The momenta $\pi(x, \mu)$ of the molecular dynamics (MD) are introduced, as elements of the Lie algebra, $\pi(x, \mu)=i \pi^{a}(x, \mu) T^{a}$. The MD Hamiltonian is finally written as $H=H_{\pi}+H_{g}+H_{f}$ with:

$$
\begin{aligned}
& H_{\pi}=\frac{1}{2} \sum_{x, \mu} \operatorname{tr}\left[\pi(x, \mu)^{2}\right]=\frac{T_{F}}{2} \sum_{x, \mu, a} \pi^{a}(x, \mu)^{2}, \\
& H_{g}=-\frac{\beta}{2 N} \sum_{x, \mu \neq v} \operatorname{tr} \mathscr{P}_{\mu v}(x) \\
& H_{f}=\sum_{x, i} \alpha_{i}^{n_{f}} \phi^{\dagger}(x)\left[Q^{2}-\beta_{i}^{\left(n_{f}\right)}\right]^{-1} \phi(x) .
\end{aligned}
$$

Also multiple pseudofermion actions and even-odd preconditioning are implemented; for details see [15].

MD evolution equations. Denoting with $\tau$ the MD time, the evolution equations for the MD Hamiltonian are:

$$
\frac{d}{d \tau} U(x, \mu)=\pi(x, \mu) U(x, \mu), \quad \frac{d}{d \tau} \pi^{a}(x, \mu)=F_{g}^{a}(x, \mu)+F_{f}^{a}(x, \mu),
$$


where the $F_{g}$ is the usual gauge force, while $F_{f}$ is the usual fermions force where all the link-variables and the generators are replaced with the corresponding quantities in the representation $R$ (see [15] for explicit computation):

$$
\begin{aligned}
& F_{g}^{a}(x, \mu)= \frac{\beta}{T_{F} N} \operatorname{Re} \operatorname{tr}\left[i T^{a} U(x, \mu) V(x, \mu)^{\dagger}\right], \\
& F_{f}^{a}(x, \mu)=-\frac{1}{T_{F}} \operatorname{Re} \operatorname{tr}\left\{i T_{R}^{a} U_{R}(x, \mu) \gamma_{5}\left(1-\gamma_{\mu}\right) \times\right. \\
&\left.\quad \times \sum_{i} \alpha_{i}^{\left(n_{f}\right)}\left[\eta_{i}(x+\hat{\mu}) \xi_{i}(x)^{\dagger}+\xi_{i}(x+\hat{\mu}) \eta_{i}(x)^{\dagger}\right]\right\},
\end{aligned}
$$

where $V(x, \mu)$ is the staple in the link $(x, \mu)$ and the pseudofermions $\eta_{i}$ and $\xi_{i}$ are defined as $\eta_{i}=\left[Q^{2}-\beta_{i}^{\left(n_{f}\right)}\right]^{-1} \phi$, and $\xi_{i}=Q \eta_{i}$. The Eqs. (2.8) are integrated using a second order Omelyan integrator [16]; moreover the exponentiation of the momenta is approximated with an exact unitary matrix [17].

Random updates. Before each MD trajectory, the momenta and the pseudofermions must be chosen with the random distributions defined by the partition function. In particular each component of the momenta is independently generated with Gaussian distribution proportional to $\exp \left(-T_{F} \pi^{a}(x, \mu)^{2} / 2\right)$. While $\phi$ is obtained by generating a pseudofermion $\tilde{\phi}$ with distribution proportional to $\exp \left(-\tilde{\phi}^{\dagger} \tilde{\phi}\right)$, and defining:

$$
\phi=Q^{-n_{f} / 2} \tilde{\phi}=\left(Q^{2}\right)^{-n_{f} / 4} \tilde{\phi} \simeq \sum_{i} \alpha_{i}^{\left(n_{f} / 2\right)}\left[Q^{2}-\beta_{i}^{\left(n_{f} / 2\right)}\right] \tilde{\phi}
$$

Metropolis test. In order to correct the error introduced by the numeric integration of the MD evolution equations, the new configuration generated by the MD evolution is accepted/rejected with a standard Metropolis test.

\section{The lattice simulations}

We simulated SU(2) gauge theory with two Dirac fermions in the adjoint representation, on $16 \times 8^{3}$ lattices, at fixed $\beta=2.0$ and various $\kappa$.

In order to check that the Dirac operator has no exceptionally small eigenvalues, we have monitored the distribution of the lowest eigenvalue of $Q^{2}$ (Fig. 3). The results obtained with fundamental fermions in [18] suggest that the width of the distribution is proportional to $a / \sqrt{V}$, therefore the existence of exceptionally small eigenvalues is suppressed in the limit of infinite volume or zero lattice spacing (at fixed value of $\kappa$ ). On the other hand at fixed volume and lattice spacing the probability of getting exceptionally small eigenvalues is non-zero if we go close enough to the chiral limit. This region of parameter space is also likely to be affected by the presence of an Aoki phase around the chiral point, which is expected to break the flavour symmetry with pattern $\mathrm{SO}(4) \rightarrow \mathrm{U}(1) \times \mathrm{U}(1)$ in the theory under scrutiny [19]. Also the Aoki phase is a lattice artifact and it is expected to vanish in the continuum limit. It is clear that even if we are observing neither exceptionally small eigenvalues nor signatures of the Aoki phase, if we are too close to the chiral limit our results may be affected by large systematic error. The only way to get reliable results is 
to check their stability while increasing the volume and reducing the lattice spacing. This has not been done in this work, in which we present data at fixed volume and fixed lattice spacing, and it remains one of our main goals for the future.

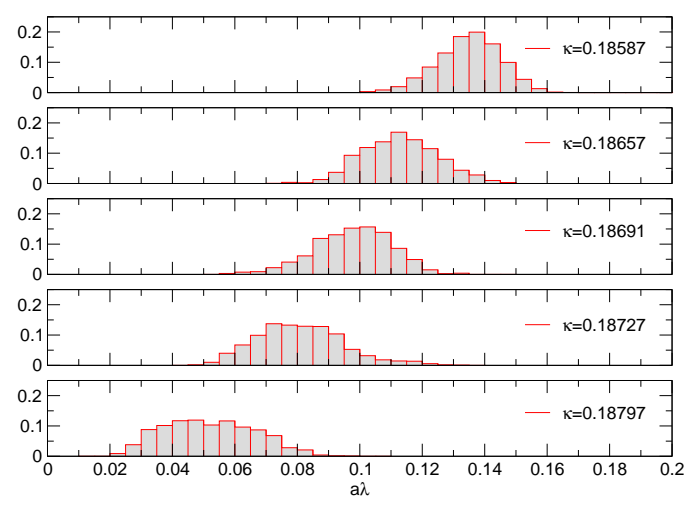

Figure 1: Probability distributions of the lowest eigenvalue of $Q^{2}$.

We measured the PCAC mass $m$ of the quarks, the pseudoscalar meson mass $M_{P S}$, its decay constant $F_{P S}$, the vectorial meson mass $M_{V}$, and the axial meson mass $M_{A}$. The results are listed in Table 1. The masses are extracted by fitting the plateau of the effective mass extracted from the correlator. On the lattices considered so far this plateau consists only of few points, and this is another source of systematic error.

\begin{tabular}{ccccccc}
$\kappa$ & $a m$ & $a M_{P S}$ & $a M_{P S}^{2} / m$ & $a F_{P S}$ & $a M_{V}$ & $M_{A} / M_{V}$ \\
\hline 0.18587 & $0.2209(30)$ & $1.149(11)$ & $5.98(14)$ & $0.399(14)$ & $1.269(12)$ & $1.51(22)$ \\
0.18657 & $0.1874(40)$ & $1.044(18)$ & $5.82(20)$ & $0.354(20)$ & $1.163(20)$ & $1.75(26)$ \\
0.18692 & $0.1559(22)$ & $0.919(13)$ & $5.41(15)$ & $0.300(11)$ & $1.029(14)$ & $1.45(7)$ \\
0.18727 & $0.1236(25)$ & $0.764(20)$ & $4.72(25)$ & $0.247(12)$ & $0.850(25)$ & $1.10(10)$ \\
0.18748 & $0.1012(20)$ & $0.652(21)$ & $4.20(27)$ & $0.215(10)$ & $0.733(22)$ & $1.00(10)$ \\
0.18769 & $0.0720(17)$ & $0.485(28)$ & $3.26(37)$ & $0.184(7)$ & $0.511(34)$ & $0.75(12)$ \\
0.18797 & $0.0238(8)$ & $0.278(25)$ & $3.26(59)$ & $0.102(8)$ & $0.275(32)$ & $0.69(17)$ \\
\hline
\end{tabular}

Table 1: Fitted values for the masses and decay constant in lattice units, in various interesting combinations.

The chiral point can be identified by fitting the PCAC mass with a linear function of $1 / \kappa$. This fit is reasonable (see Fig. 3) and yields $\kappa_{c}=0.18827(4)$.

If the standard scenario of the chiral symmetry breaking were realized, then the ratio $a M_{P S}^{2} / m$ should become a constant in the chiral limit. Although it goes flat in the two lightest points (see Table 1), it has an unexpected behaviour, dropping rapidly from the heaviest points and then stopping abruptly. Robust conclusions can be drawn in this case and a more careful study of the chiral limit is required.

On the contrary, the linear behaviour of the PS decay constant with the PCAC mass is pretty clear. A linear extrapolation can be made using all the points and the result for the chiral limit is $a F_{P S}(m=0)=0.084(4)$ (Fig. 3).

The linear behaviour of the V mass with the PCAC mass is quite good but it is not clear if the vectorial meson stays massive in the chiral limit (Fig. 3). The ratio $M_{V} / M_{P S}$ is also plotted in 

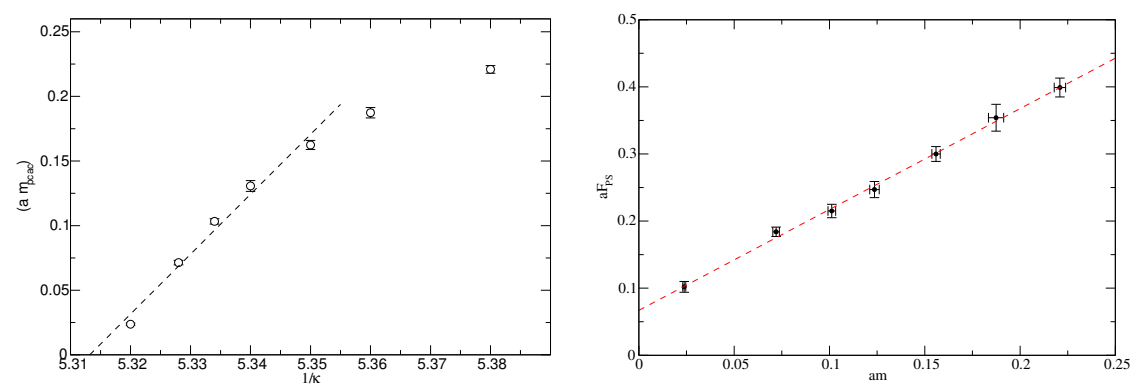

Figure 2: On the left side, linear extrapolation of the PCAC mass as a function of the inverse hooping parameter $1 / \kappa$. Only the five lowest points are used for the fit. On the right side, linear extrapolation of the PS decay constant as a function of the PCAC mass.

Fig. 3 ; in the chiral symmetry were spontaneously broken, this ratio should diverge in the chiral limit, while we observe here that it stays finite (and compatible with 1).
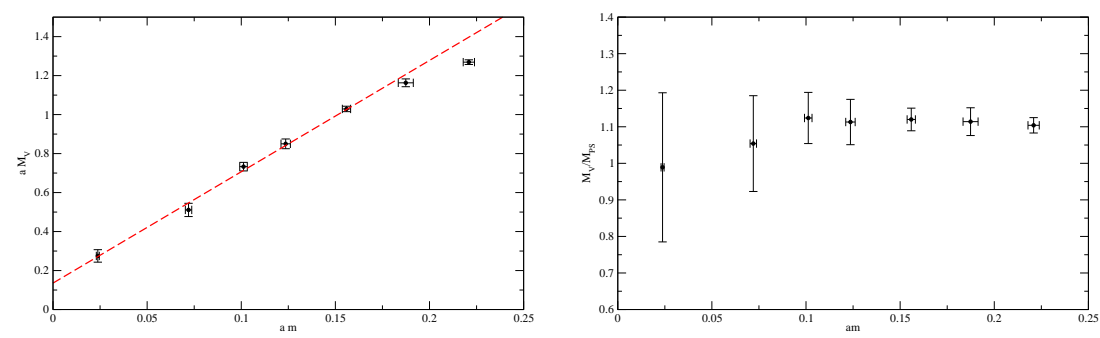

Figure 3: On the left, linear extrapolation of the vectorial meson mass as a function of the PCAC mass. On the right, the ratio $M_{V} / M_{P S}$ as a function of the PCAC mass.

Finally the ratio $M_{A} / M_{V}$ is reported in the last column of Table 1 . The lightest points seems to indicate an inversion of the vectorial and axial mesons, and therefore a violation of the second Weinberg sum rule, which is another hint for non-standard physics.

\section{Conclusions}

We presented an implementation of the RHMC algorithm for Wilson fermions in a generic representation of the gauge group $\mathrm{SU}(N)$. We developed a bespoke code - HiRep - and we used it to obtain some preliminary results for the $\mathrm{SU}(2)$ theory with two Dirac fermions in the adjoint representation, on a $16 \times 8^{3}$ lattice at fixed $\beta=2.0$. Since we know very few about this theory, we chose to proceed with caution in interpreting the results. We pointed out that some results seem to fit in the standard scenario of a QCD-like theory (the linear extrapolation of the PCAC mass with $1 / \kappa$, the linear extrapolation of the PS decay constant and of the vectorial meson mass with the PCAC mass), some other results need more investigation to be understood (the behaviour of the ratio $a M_{P S}^{2} / m$ ), other results seem to indicate non-standard phenomena (the ratio $M_{V} / M_{P S}$ or the inversion of the axial and vectorial meson masses). Finally we stressed the importance of checking the stability of these results by increasing the volume and reducing the lattice spacing, especially in a region close to the chiral limit. 


\section{References}

[1] S. Weinberg, Implications of dynamical symmetry breaking, Phys. Rev. D13 (1976) 974-996.

[2] L. Susskind, Dynamics of spontaneous symmetry breaking in the Weinberg-Salam theory, Phys. Rev. D20 (1979) 2619-2625.

[3] C. T. Hill and E. H. Simmons, Strong dynamics and electroweak symmetry breaking, Phys. Rept. 381 (2003) 235-402 [hep-ph/0203079].

[4] F. Sannino, Dynamical stabilization of the fermi scale: Phase diagram of strongly coupled theories for (minimal) walking technicolor and unparticles [0804.0182 [hep-ph]].

[5] E. Corrigan and P. Ramond, A note on the quark content of large color groups, Phys. Lett. B87 (1979) 73.

[6] A. Armoni, M. Shifman and G. Veneziano, Exact results in non-supersymmetric large N orientifold field theories, Nucl. Phys. B667 (2003) 170-182 [hep-th/0302163].

[7] T. Banks and A. Zaks, On the phase structure of vector-like gauge theories with massless fermions, Nucl. Phys. B196 (1982) 189.

[8] D. D. Dietrich and F. Sannino, Conformal window of $S U(N)$ gauge theories with fermions in higher dimensional representations, Phys. Rev. D75 (2007) 085018 [hep-ph / 0611341 ].

[9] R. Foadi, M. T. Frandsen, T. A. Ryttov and F. Sannino, Minimal walking technicolor: Set up for collider physics, Phys. Rev. D76 (2007) 055005 [0706.1696 [hep-ph]].

[10] S. Catterall and F. Sannino, Minimal walking on the lattice, Phys. Rev. D76 (2007) 034504 [0705.1664 [hep-lat]].

[11] T. Appelquist, G. T. Fleming and E. T. Neil, Lattice study of the conformal window in QCD-like theories [0712.0609 [hep-ph]].

[12] Y. Shamir, B. Svetitsky and T. DeGrand, Zero of the discrete beta function in SU(3) lattice gauge theory with color sextet fermions [0803.1707 [hep-lat]].

[13] A. Deuzeman, M. P. Lombardo and E. Pallante, The physics of eight flavours [0804.2905 [hep-lat]].

[14] M. A. Clark and A. D. Kennedy, Accelerating dynamical fermion computations using the rational hybrid monte carlo (RHMC) algorithm with multiple pseudofermion fields, Phys. Rev. Lett. 98 (2007) 051601 [hep-lat/0608015].

[15] L. Del Debbio, A. Patella and C. Pica, Higher representations on the lattice: numerical simulations. SU(2) with adjoint fermions [0805.2058 [hep-lat]].

[16] T. Takaishi and P. de Forcrand, Testing and tuning new symplectic integrators for hybrid Monte Carlo algorithm in lattice QCD, Phys. Rev. E73 (2006) 036706 [hep-lat/ 0505020 ].

[17] M. Luscher, Schwarz-preconditioned HMC algorithm for two-flavour lattice QCD, Comput. Phys. Commun. 165 (2005) 199-220 [hep-lat/ 0409106 ].

[18] L. Del Debbio, L. Giusti, M. Luscher, R. Petronzio and N. Tantalo, Stability of lattice QCD simulations and the thermodynamic limit, JHEP 02 (2006) 011 [hep-lat / 0512021 ].

[19] L. Del Debbio, M. T. Frandsen, H. Panagopoulos and F. Sannino, Higher representations on the lattice: perturbative studies [0802.0891 [hep-lat]]. 